Noname manuscript No.

(will be inserted by the editor)

\title{
A Cost-based Storage Format Selector for Materialized Results in Big Data Frameworks
}

\author{
Rana Faisal Munir ${ }^{1,2}$. Alberto Abelló ${ }^{1}$. \\ Oscar Romero ${ }^{1}$ - Maik Thiele ${ }^{2}$. \\ Wolfgang Lehner ${ }^{2}$
}

Received: date / Accepted: date

\begin{abstract}
Modern big data frameworks (such as Hadoop and Spark) allow multiple users to do large-scale analysis simultaneously, by deploying DataIntensive Workflows (DIWs). These DIWs of different users share many common tasks (i.e, 50-80\%), which can be materialized and reused in future executions. Materializing the output of such common tasks improves the overall processing time of DIWs and also saves computational resources. Current solutions for materialization store data on Distributed File Systems by using a fixed storage format. However, a fixed choice is not the optimal one for every situation. Specifically, different layouts (i.e., horizontal, vertical or hybrid) have a huge impact on execution, according to the access patterns of the subsequent operations.

In this paper, we present a cost-based approach that helps deciding the most appropriate storage format in every situation. A generic cost-based framework that selects the best format by considering the three main layouts is presented. Then, we use our framework to instantiate cost models for specific Hadoop storage formats (namely SequenceFile, Avro and Parquet), and test it with two standard benchmark suits. Our solution gives on average $1.33 \mathrm{x}$
\end{abstract}

Rana Faisal Munir

E-mail: fmunir@essi.upc.edu

Alberto Abelló

E-mail: aabello@essi.upc.edu

Oscar Romero

E-mail: oromero@essi.upc.edu

Maik Thiele

E-mail: maik.thiele@tu-dresden.de

Wolfgang Lehner

E-mail: wolfgang.lehner@tu-dresden.de

${ }^{1}$ Universitat Politècnica de Catalunya (UPC), Barcelona, Spain

${ }^{2}$ Technische Universität Dresden (TUD), Dresden, Germany

This is a post-peer-review, pre-copyedit version of an article published in Distributed and parallel databases. The final authenticated version is available online at: http://dx.doi.org/10.1007/s10619-019-07271-0 
speedup over fixed SequenceFile, 1.11x speedup over fixed Avro, 1.32x speedup over fixed Parquet, and overall, it provides $1.25 \mathrm{x}$ speedup.

Keywords Big Data · Data-Intensive Workflows · Materialized Results · Storage Format · HDFS · Cost Model

\section{Introduction}

Data analysis plays a decisive role in today's data-oriented organizations, which produce and store large volumes of data (i.e., in the order of petabytes to zettabytes [26]). To store and process such data, organizations typically rely on the use of distributed frameworks, such as Apache Hadoop ${ }^{1}$ and Apache Spark $^{2}$. These frameworks are used by multiple users and the data is processed by deploying complex analytical workflows that orchestrate multiple tasks. Each task produces an output that is used as input for the subsequent tasks. The workflows may have many redundant tasks, whose output, if materialized, can be reused to improve the overall execution time. In this paper, we refer to the materialization of the output of redundant tasks as Materialized Result (MR).

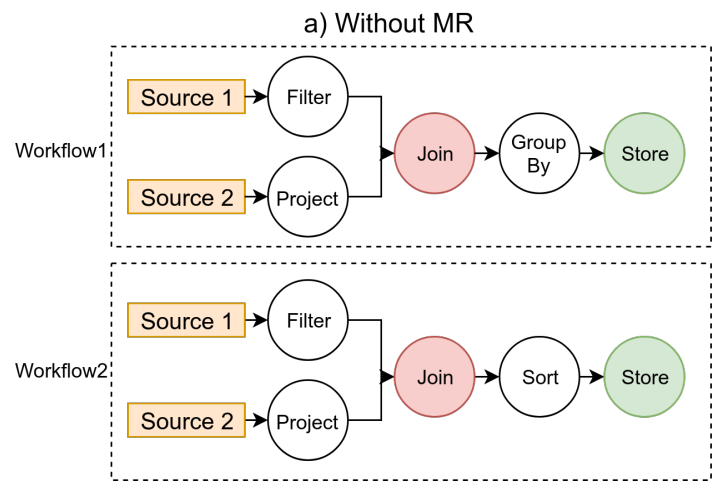

b) With MR

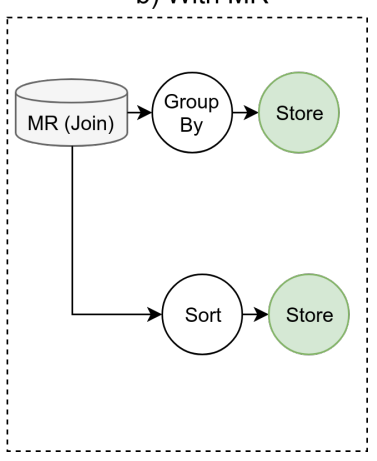

Fig. 1 An example of a Materialized Result (MR)

Figure 1a shows two analytical workflows, orchestrating multiple tasks. They both have a common task (i.e., JOIN), which can be materialized for reuse. That is, if these workflows were to be submitted again as shown in Figure $1 \mathrm{~b}$, they would not require to recompute the JOIN, because that would have already been materialized and could be reused by both. This $M R$ would help on saving computational resources and reducing the execution time. Yet, note that MRs are different from intermediate results (IRs), produced by different tasks in the same workflow. For instance, in Spark, the IRs are always

\footnotetext{
${ }^{1}$ http://hadoop.apache.org

${ }^{2}$ https://spark.apache.org
} 
stored in memory and discarded afterwards. Whereas, a $M R$ is stored in the disk and its purpose is to be reused not only by the same workflow but also by different workflows.

The importance of MRs has been acknowledged in an in-depth study of seven enterprises [6], where it was shown that $80 \%$ of their different analytical workflows had redundant/common tasks. Similarly, recent studies $[14,15]$ from Microsoft have shown that $65 \%$ of their workflows have redundant parts. These studies imply that a proper management of $M R s$ could provide benefits in terms of computational resources and execution time. Yet, a solution to this problem means answering the following questions: "(1) which MR should be chosen?" and "(2) which layout should be used for its storage?".

Answers to the first question have already been given. Several approaches $[8,14,15,19,23,27]$, help on choosing the MRs that minimize the overall analytical workflow execution times. However, $M R s$ are typically stored in a Distributed File System (DFS), using a single fixed layout, thus, ignoring the second question of "which layouts should be used when persisting MRs?". The importance of the such question lies on the fact that, since DFS I/O operations are expensive, the analytical workflow execution times can be further reduced by choosing the physical storage layouts based on the operators. Obviously, a fixed storage layout can not be optimal for all types of workloads. Indeed, [1] shows the importance of storing data according to their access pattern and that single fixed layouts are not good for all types of workloads. Similarly, $[11,16]$ also focus on the importance of storing data according to their access patterns and highlight the effect of different storage layouts on different workloads $^{3}$. Nevertheless, no current solution is able to choose the layout of MRs automatically.

In this paper, we present a cost-based approach to address the second question and find the most appropriate storage layout for $M R s$. However, since a cost model requires statistical information about the data and the analytical workflows in order to make a decision, we also propose the use of a rulebased approach for cold-start. Therefore, we first apply rules for choosing storage layouts, while collecting the statistical information. Once the required statistical information has been gathered, we can apply the proposed costmodel.

Our contributions are as follows:

- We present a generic I/O model for the three main layouts (i.e., horizontal, vertical, and hybrid) in big data frameworks, for estimating their read and write costs.

- We instantiate the cost model on Hadoop Distributed File System (HDFS), for SequenceFile, Avro, and Parquet.

- We propose and implement a generic framework for big data systems, to store the selected $M R s$ in the appropriate storage format.

- We conduct comprehensive experiments on two de-facto standard industry benchmarks for Decision Support Systems (DSSs). The results show that

\footnotetext{
${ }^{3}$ http://www.svds.com/how-to-choose-a-data-format
} 
our approach reduces the overall workflow execution times when compared to using single fixed layouts, by providing a $1.25 \mathrm{x}$ average speedup.

The remainder of this paper is organized as follows: In Section 2, we discuss the storage layouts and our motivation. In Sections 3 and 4, we discuss our approach and the generic cost model in detail. In Section 5, we report on our experimental results. In Section 6, we discuss the related work. Finally, in Section 7 , we conclude the paper.

\section{Background and Motivation}

In this section, we discuss the different storage layouts available and exemplify them with their corresponding instantiation for HDFS. Moreover, we discuss existing materialized solutions and also motivate our work by illustrating the fixed layout limitations.

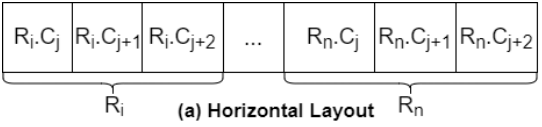

Fig. 2 Horizontal and vertical layouts

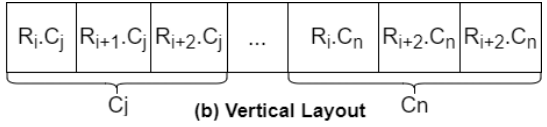

\subsection{Storage layouts}

There are many layouts, used in different processing frameworks, that can be divided into three categories based on how they fragment data: horizontal, vertical or hybrid. Each concrete layout has its own physical storage structure that is beneficial for a specific kind of workloads.

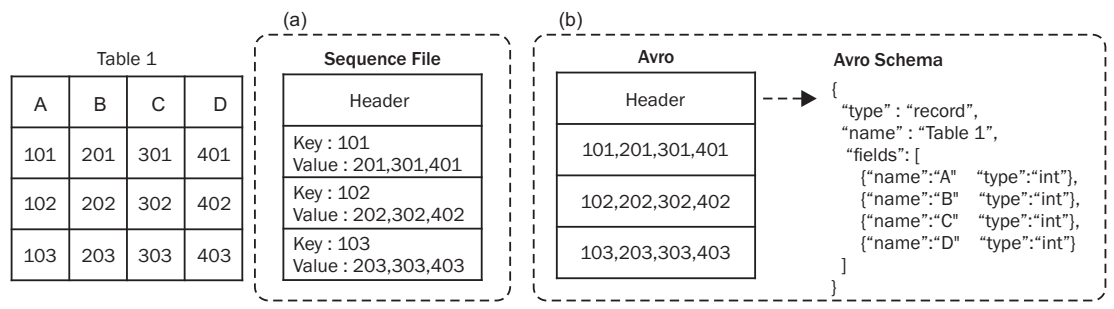

Fig. 3 Examples of SequenceFile and Avro layouts 


\subsubsection{Horizontal layouts}

They are organized row-wise, and the attributes of each row are stored together, as shown in Figure 2a (where $R$ represents the row and $C$ represents the column of a row). For this reason, a horizontal layout especially suits scan-based workloads. However, if a query is just referring to a small subset of columns, this layout results in a low effective read ratio, since non-required columns will be fetched anyway. In HDFS, the horizontal layout is implemented by SequenceFile ${ }^{4}$ and $\mathrm{Avro}^{5}$. SequenceFile is a special type of horizontal layout storing simple key-value data, whereas Avro explicitly splits data into columns inside every row. In other words, it embeds schema information. Figure 3 shows an example of a table and its corresponding format in SequenceFile (i.e., Figure 3a) and Avro (i.e., Figure 3b).

\begin{tabular}{|c|c|c|r|}
\multicolumn{1}{c}{ Table 1} \\
\hline A & B & C & D \\
\hline 101 & 201 & 301 & 401 \\
\hline 102 & 202 & 302 & 402 \\
\hline 103 & 203 & 303 & 403 \\
\hline
\end{tabular}

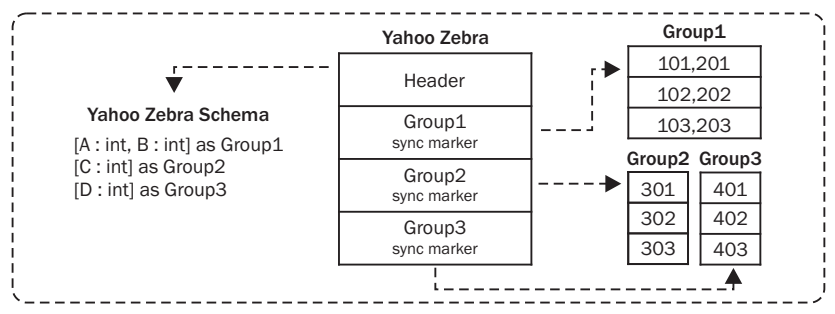

Fig. 4 Example of Zebra layout

\subsubsection{Vertical layouts}

They divide each row into columns, and store each column separately, which is beneficial for workloads reading just few columns. Thus, these layouts excel in projection-based workloads. Figure $2 \mathrm{~b}$ sketches the physical structure of vertical layouts. Zebra ${ }^{6}$, illustrated in Figure 4, is an implementation of this kind for HDFS. Zebra also allows to group columns together, but without any horizontal partition.

\subsubsection{Hybrid layouts}

They are a combination of horizontal and vertical layouts, having two alternative implementations: Either the data is divided horizontally and then vertically, like in Figure 5a, or vice versa, like in Figure 5b. Both cases are especially helpful for combinations of projection and selection operations. There are many implementations of this kind, but the most popular ones in HDFS are

\footnotetext{
${ }^{4}$ https://wiki.apache.org/hadoop/SequenceFile

${ }^{5}$ https://avro.apache.org

${ }^{6}$ https://wiki.apache.org/pig/zebra
} 


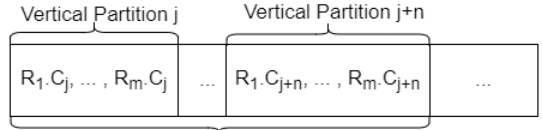

Horizontal Partition $m$

(a)

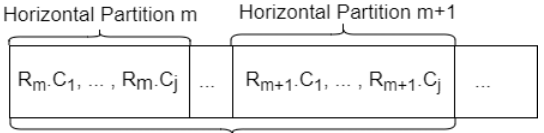

Vertical Partition j

(b)

Fig. 5 Hybrid layouts

Optimized Row Columnar (ORC) $)^{7}$ and Parquet $^{8}$, both primarily fragmenting data horizontally. Figure 6 exemplifies Parquet.

\begin{tabular}{|c|c|c|c|}
\hline \multicolumn{4}{|c|}{ Table 1} \\
\hline A & B & C & D \\
\hline 101 & 201 & 301 & 401 \\
\hline 102 & 202 & 302 & 402 \\
\hline 103 & 203 & 303 & 403 \\
\hline
\end{tabular}

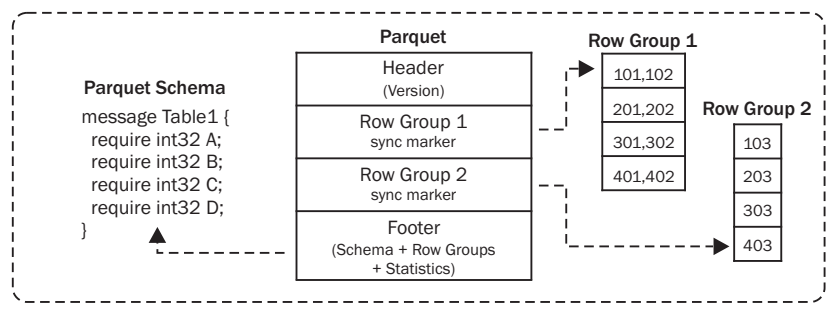

Fig. 6 Example of Parquet layout

\subsection{Existing materialized solutions}

As discussed previously, there are many available materialization solutions $[8$, $14,19,23,27]$ for big data frameworks, which can be used to select MRs. In this paper, we use ReStore [8] because it is a simple but powerful solution based on heuristic rules. Importantly, there is an available implementation ${ }^{9}$ of ReStore in Apache $\mathrm{Pig}^{10}$, which we used for our experiments. However, our approach is not tied to any materialization solution and ReStore could be replaced by other more sophisticated methods $[14,15,21]$ if required. The heuristics of ReStore are categorized into conservative and aggressive: Conservative heuristics aim at materializing the outputs of those operators, i.e., Projection and Selection, which reduce the size of the data. Whereas, aggressive heuristics materialize the outputs of those operators, i.e., Join, Group By, which are computationintensive. Section 5 discusses the results of ReStore for $\mathrm{TPC}-\mathrm{H}^{11}$ and $\mathrm{TPC}-$ $\mathrm{DS}^{12}$.

\footnotetext{
${ }^{7}$ https://orc.apache.org

${ }^{8}$ http://parquet.apache.org

${ }^{9}$ https://github.com/ami07/ReStoreV2

${ }^{10}$ https://pig.apache.org

${ }^{11}$ http://www.tpc.org/tpch

12 http://www.tpc.org/tpcds
} 

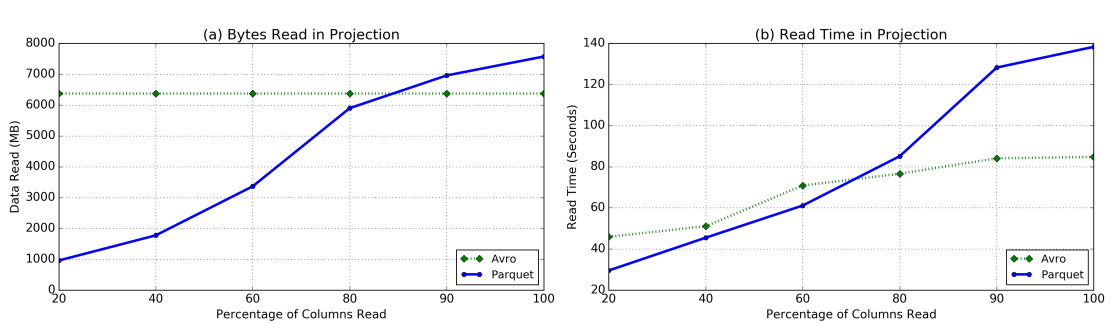

Fig. 7 The effect of the number of retrieved columns on different layouts

\subsection{Layout performance comparison}

Ad-hoc and exploratory analysis are very popular among data analysts, helping them to understand different aspects of their business. However, it is very difficult to tune a system for such scenarios since the workload is very dynamic, and current solutions are not considering layouts depending on workflow operations, and ignore this fact when storing MRs in the disk.

To illustrate the drawback of the current static approaches let us assume the following example from TPC-H. Lets assume the join between Lineitem and Part tables is chosen as a $M R$. Figure 7 shows the execution time of a simple projection-based query for horizontal and hybrid layouts. It can be seen that Parquet (i.e., a hybrid layout) performs well when the total amount of data read from disk is below 75\%, whereas Avro (i.e., a horizontal layout) performs better as soon as we read more than $75 \%$ of data. Thus, this shows that the characteristics of the query/workflow help to determine the optimal layout.

\section{Our Approach in a Nutshell}

From here on, a Data-Intensive Workflow (DIW) is represented as a directedacyclic graph of operations (an example can be seen in Figure 12). Nodes represent operations and directed edges show the dependencies between the nodes. The starting node of an edge produces the data to be consumed by the ending node (note that a node output can be consumed by several nodes). Different DIWs can have multiple common nodes, whose output when materialized is referred to as Materialized Results (MRs).

Given a DIW, Figure 8 illustrates the flowchart of our approach. Following the two questions introduced in Section 1, first, (i) it selects MRs using ReStore and, then, (ii) for each of them, it chooses the best storage layout.

\subsection{Storage layout selection}

Since existing materialized solutions use a fixed layout for $M R s$, our approach helps them to decide the best storage layout for each chosen $M R$. If statistical 


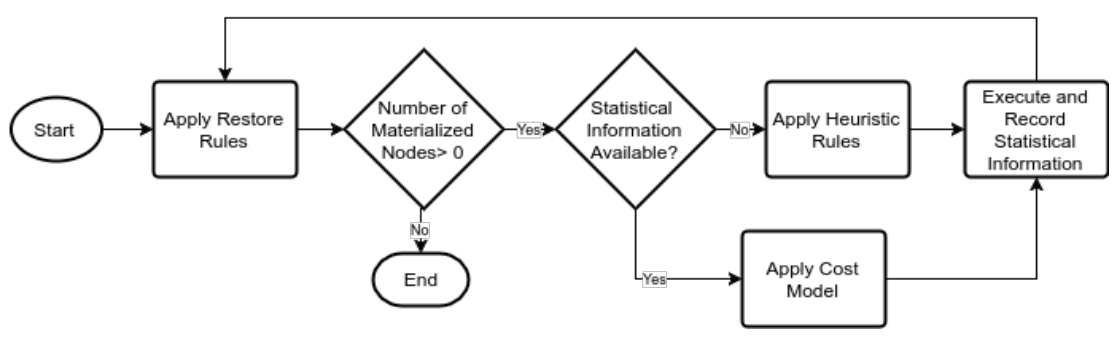

Fig. 8 Flowchart of our approach

information about $M R s$ is available, we use the cost-based model to decide the storage layout. If for any reason we have not enough statistical information (see the variables required by our cost model in Section 4) to make a decision on a given $M R$, we can still apply heuristic rules [22] to determine the storage layout. The heuristic rules choose a storage layout based only on the operation type. Obviously, it might happen that the heuristic rules do not choose the best storage layouts since they do not consider essential information to estimate the total volume of data to be read from the disk. For example, projections/selections can perform differently based on the percentage of columns/rows read. Thus, factors such as the number of columns and selectivity factors may drastically impact on the operation performance depending on the storage layouts, as illustrated in Figure 7. These factors cannot be considered in heuristic rules, because heavily depend on the concrete operation and data characteristics. Still, heuristic rules provide a fair first-approach to the problem with small computational requirements in scenarios where there is lack of information. Oppositely, if the required statistics are available, the cost-based approach, like the one in Section 4, is more accurate. Finally, the DIW is executed and the chosen MRs are stored with their chosen storage formats. Our approach also records/updates the needed statistical information to be used in the future.

\section{Cost-Based Model}

The cost-based model relies on a wide range of statistical information that is summarized in Table 1, containing system constants, data statistics, workload statistics as well as layout variables. The system constants are generally based on [16]. We only extended the list with specific variables related to the selectivity factor and storage layouts. We assume the constants depending on the configuration of the environment (e.g., $B W_{\text {disk }}, B W_{n e t}$ ) are given and the statistics are collected during the DIW execution. Moreover, it should be noted that we consider only I/O cost in our cost model, because it is the dominant factor in DIWs.

\footnotetext{
${ }^{13}$ Extra 4 bytes are considered for variable length columns
} 
Table 1 Parameters of the Cost Model

\begin{tabular}{|c|c|}
\hline Variable & Description \\
\hline \multicolumn{2}{|r|}{ System Constants } \\
\hline$R$ & Replication factor \\
\hline$p$ & Probability of accessed replica being local \\
\hline Size $($ Chunk $)$ & Block size in the DFS \\
\hline$B W_{d i s k}$ & Disk bandwidth \\
\hline$B W_{n e t}$ & Network bandwidth \\
\hline Time $_{\text {seek }}$ & Disk seek time \\
\hline \multirow{2}{*}{ Time $_{\text {disk }}$} & Size(Chunk) \\
\hline & $B W_{d i s k}$ \\
\hline \multirow{2}{*}{ Time $_{n e t}$} & Size(Chunk) \\
\hline & $B W_{\text {net }}$ \\
\hline \multicolumn{2}{|r|}{ Data Statistics } \\
\hline$|M R|$ & Number of Rows in MR \\
\hline Size (Row) & Average Row Size of MR \\
\hline $\operatorname{Size}(\mathrm{Col})^{13}$ & Average Column Size of MR \\
\hline $\operatorname{Cols}(M R)$ & Columns of MR \\
\hline \multicolumn{2}{|r|}{ Workload Statistics } \\
\hline $\operatorname{RefCols}(M R)$ & Number of columns used in an operation \\
\hline$S F$ & Selectivity factor of an operation \\
\hline \multicolumn{2}{|r|}{ Layout Variables } \\
\hline Size(RowGroup) & Row group size of hybrid layouts \\
\hline Size $($ Meta layout $)$ & Meta data size for a given layout \\
\hline Size $($ Bodylayout $)$ & Size of the body of a layout \\
\hline Size $\left(\right.$ Header layout $\left._{\text {lay }}\right)$ & Size of the header of a layout \\
\hline Size (Footer layout $)$ & Size of the footer of a layout \\
\hline Used $_{\text {chunks }}($ Layout $)$ & Number of chunks of a layout \\
\hline $\operatorname{Used}_{R G}($ Layout $)$ & Number of row group of hybrid layouts \\
\hline Used rows $_{\text {(RowGroup })}$ & Number of rows of a row group \\
\hline Seeks (Layout) & Total number of seeks for a given layout \\
\hline
\end{tabular}

$$
\begin{aligned}
\operatorname{Size}(\text { Layout }) \quad & \text { Size }\left(\text { Header }_{\text {layout }}\right) \\
& + \text { Size }\left(\text { Body }_{\text {layout }}\right) \\
& + \text { Size }\left(\text { Footer }_{\text {layout }}\right) \\
\text { Used }_{\text {chunks }}(\text { Layout })= & \frac{\text { Size }(\text { Layout })}{\text { Size }(\text { Chunk })} \\
\operatorname{Seeks}(\text { Layout }) \quad= & \text { Used } \left._{\text {chunks }}(\text { Layout })\right\rceil
\end{aligned}
$$

Independently of the kind of layout, the driving factor of our cost model is the file size. The body, together with the header and footer compose it (Equation 1). From that, we can obtain the number of chunks used (Equation 2) and the number of disk seeks we need to reach them (Equation 3). The number of seeks is equal to the total number of chunks rounded up, because one seek is required for every chunk, even if it is not full. Note that modern Solid State Disks (SSDs) also have seek time (i.e., time required to turn on the right 
circuit), however their seek time is much less (i.e., 0.1ms) compared to hard disks (i.e., $0.8 \mathrm{~ms})^{14}[20]$. Thus, our cost model still applies and we would only need to update the system constants accordingly.

In the next subsections, we analyze the cost of data writes and reads, because they are the dominant factors in the overall execution time of DIWs. The write cost model estimates the data volume footprint of each layout as well as the cost incurred in writing it, while the read cost model estimates the cost of an operation depending on the access pattern. Regarding the latter, given the simplicity of a file system (far from that of a DBMS) only three operations are possible (namely full scan, projection, and selection).

$$
\begin{aligned}
W_{\text {WriteTransfer }}= & \frac{\text { Time }_{\text {disk }}+(R-1) * \text { Time }_{\text {net }}}{\text { Time }_{\text {seek }}+\text { Time }_{\text {disk }}+(R-1) * \text { Time }_{\text {net }}} \\
\text { Cost }_{\text {write }}(\text { Layout })= & \text { Used }_{\text {chunks }}(\text { Layout }) * W_{\text {WriteTransfer }} \\
& +\operatorname{Seeks}_{\text {Layout }} *\left(1-W_{\text {WriteTransfer }}\right)
\end{aligned}
$$

\subsection{Write cost}

First of all, we have to take into consideration that distributed processing frameworks are using DFS to store data into multiple chunks. Thus, the number of chunks of a file is used to estimate the overall writing costs. Given that a chunk consists of multiple contiguous disk blocks and inside it, sequential read is guaranteed, assuming that the chunk size is smaller than a disk cylinder, the write cost can be simply computed as the number of chunks plus the seek cost to locate the position of each. Nevertheless, since our cost model is thought for distributed processing frameworks, we further need to consider the replication factor $R$ used for fault-tolerance, and therefore the network costs for writing $R$ copies needs to be taken into account. We assume that the replication procedure is sequential (as it is in HDFS) and the multiple copies are written one after another. Equation 4 gives the weight of transferring a chunk by considering the network and the disk write against the seek costs. Finally, Equation 5 shows the total write cost taking both seek and transfer weights into account.

In the following, we present the write cost for each horizontal, vertical and hybrid layouts.

\subsubsection{Horizontal layouts}

They store data row-wise into the body section. Oppositely, metadata containing information such as schema and version, is written into the header and footer sections. Nevertheless, in some implementations, additional metadata is also written in the body with every row, for example, metadata used

${ }^{14} \mathrm{http} / /$ www.ieee802.org/3/CU4HDDSG/public/sep15/Kipp_CU4HDDsg_01a_0915.pdf 
to separate each row or each column (i.e., its size is not constant and depends on the number of columns).

$$
\begin{aligned}
& \operatorname{Size}\left(\text { Body }_{\text {horizontal }}\right)=\operatorname{Size}\left(\operatorname{Meta}_{H \text { Body }}\right)+|M R| *\left(\operatorname{Size}_{\left(\text {Meta }_{H}\right. \text { Row }}\right) \\
& +\operatorname{Size}(\operatorname{Row}))
\end{aligned}
$$

Equation 6 estimates the size of the body by multiplying the average row size and metadata (i.e., Size $\left.\left(M e t a_{H R o w}\right)\right)$ by the total number of rows, plus other metadata (i.e., Size $\left.\left(M e t a_{H B o d y}\right)\right)$ we may find in the body section.

$$
\begin{aligned}
& \text { Size }(\text { OneCol }) \quad=\operatorname{Size}(\mathrm{Col}) *|M R| \\
& \operatorname{Size}\left(\text { Body }_{\text {vertical }}\right)=\operatorname{Size}\left(\text { Meta }_{V \text { Body }}\right)+\operatorname{Cols}(M R) *\left(\operatorname{Size}_{\left(\text {Meta }_{V C o l}\right.}\right) \\
& + \text { Size }(\text { OneCol }))
\end{aligned}
$$

\subsubsection{Vertical layouts}

They store each column independently (i.e., values of a column, which share the same data type, are stored consecutively) using a separator (i.e., Size(Meta Body)) of fixed size between columns. Equation 7 provides the estimation of the individual column size, which is used in Equation 8 to determine the overall size of the body by multiplying the size of one column by the total number of columns.

$$
\begin{aligned}
& \operatorname{Used}_{R G}(\text { Hybrid })=\frac{\left.\operatorname{Cols}(M R) *\left(\operatorname{Size}_{(\text {Meta }} \text { Col }\right)+|M R| * \operatorname{Size}(\mathrm{Col})\right)}{\operatorname{Size}(\text { RowGroup })} \\
& \operatorname{Size}\left(\operatorname{Meta}_{\text {hybrid }}\right)=\left\lceil\operatorname{Used}_{R G}(\text { Hybrid })\right\rceil * \operatorname{Size}\left(\operatorname{Meta}_{Y \text { RowGroup }}\right) \\
& \operatorname{Size}\left(\text { Bodyhybrid }_{1}\right)=\operatorname{Used}_{R G}(\text { Hybrid }) * \operatorname{Size}(\text { RowGroup }) \\
& +\operatorname{Size}(\text { Meta } \text { hybrid })
\end{aligned}
$$

\subsubsection{Hybrid layouts}

They are a combination of horizontal and vertical layouts. They divide rows into horizontal partitions known as row groups and each row in one row group is further divided into vertical partitions storing each column separately, and inserting metadata (i.e., Size $\left.\left(M e t a_{Y C o l}\right)\right)$ between them. Additionally, they also store metadata (i.e., Size (MetaY RowGroup $)$ ) for every row group. Thus, the total size of the body depends on the number of row groups being used, which can be estimated as in Equation 9 and the size of metadata of row groups is estimated in Equation 10. Notice that the metadata of the row group is stored irrespectively of it being completely full, so this must be rounded up. Furthermore, Equation 11 obtains the size of the body by multiplying the 
number of row groups by the size of a row group and by adding the total size of metadata.

4.2 Read cost

This section presents the read cost model for scan, projection and selection operations. All DIW operations in current massively distributed processing environments use a full scan access pattern on the DFS, except projection and selection operations that are specifically supported natively in some storage layouts. Thus, we consider them separately in the following.

$$
\begin{aligned}
\operatorname{Size}\left(\operatorname{Scan}_{\text {layout }}\right)= & \operatorname{Size}(\text { Layout }) \\
& +\left(\operatorname{Used}_{\text {chunks }}(\text { Layout }) * \operatorname{Size}\left(\text { Meta }_{\text {layout }}\right)\right)
\end{aligned}
$$

\subsubsection{Scan}

It reads all stored data from the disk, irrespective of the layout being used. Relatively, the metadata (such as schema, statistics, etc.) stored inside header or footer sections, reads separately in each task. The reason is that the distributed processing engines (such as Hadoop and Spark) create a separate process for each task with its own memory. This memory is not accessible to other tasks and hence, forces to read all metadata in each task separately, and consequently, increases the reading size. The number of tasks is equal to the number of used chunks. Equation 12 estimates the scan size, which can be used further to estimate the scan cost.

The scan cost purely depends on the number of used chunks to be read. Assuming the block is the transfer unit between disk and memory, there are three factors impacting the cost: the average seek time needed to locate a disk block cylinder, the rotation time to move the disk head over the cylinder to reach the block, and the transfer time to bring data in the block from disk into memory. Nevertheless, despite every chunk consists of multiple blocks on disk, it should be noted that DFS typically guarantee that all disk blocks are contiguous within one disk cylinder, under the assumption that the chunk size does not go beyond the cylinder size. This is why we do not need to consider seek time for all the disk blocks. Instead, we only consider seek time once for every chunk. Also, as confirmed in our experiments, the rotation time is negligible, because modern hardware and operating systems implement very effective pre-fetching techniques. Furthermore, our cost model is also applicable to SSDs. Since SSDs have very small seek time and high I/O speed, the corresponding system constants would simply be replaced respectively. For the rest, since the basic unit of our cost model is defined in terms of bytes, all the estimations will remain the same. 


$$
\begin{aligned}
W_{\text {ReadTransfer }}= & \frac{\text { Time }_{\text {disk }}+(1-p) * \text { Time }_{\text {net }}}{\text { Time }_{\text {seek }}+\text { Time }_{\text {disk }}+(1-p) * \text { Time }_{\text {net }}} \\
\operatorname{Used}_{\text {chunks }}\left(\text { Scan }_{\text {layout }}\right)= & \frac{\operatorname{Size}_{\left(\text {Scan }_{\text {layout }}\right)}}{\operatorname{Size}(\text { Chunk })} \\
\operatorname{Cost}_{\text {scan }}(\text { Layout }) \quad & \operatorname{Used}_{\text {chunks }}\left(\text { Scan }_{\text {layout }}\right) * W_{\text {ReadTransfer }} \\
& \left.+\operatorname{Seeks}_{\text {Layout }}\right) *\left(1-W_{\text {ReadTransfer }}\right)
\end{aligned}
$$

On the other hand, we have to take under consideration that in a distributed data processing framework data can be accessed remotely. Consequently, we introduce a probability $p$ to indicate the likelihood of chunks being accessed locally (i.e., data shipping through the network is not needed to reach the operation executor). This is used to estimate the weight of transferring the chunk data compared to the corresponding seek time using Equation 13. Then, Equation 14 estimated the total number of read chunks and Equation 15 provides the scan cost taking both the seek and the transfer cost into account with the corresponding weights.

\subsubsection{Projection}

It helps in fetching only some columns from disk (skipping others) to save some I/Os. Its cost depends on the support provided by each layout.

Horizontal layouts. They do not provide specific support for projection operation, but actually use a full scan to bring all the data into memory and only afterwards discard the unnecessary columns. Therefore, its cost is exactly the same as that of scan (i.e., Equation 15).

$$
\begin{aligned}
& \text { Size } \left.\left(\text { Project }_{\text {vertical }}\right)=\operatorname{Size}\left(\text { Header }_{\text {vertical }}\right)+\operatorname{Size}_{\left(\text {Footer }_{\text {vertical }}\right)}\right) \\
& +\operatorname{Size}(\mathrm{OneCol}) * \operatorname{RefCols}(M R)
\end{aligned}
$$

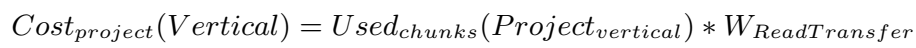

$$
\begin{aligned}
& +\operatorname{RefCols}(\mathrm{MR}) * \operatorname{Seeks}(\mathrm{OneCol}) \\
& \text { * }\left(1-W_{\text {ReadTransfer }}\right)
\end{aligned}
$$

Vertical layouts. They do support projections. Their cost depends on the size retrieved data, which is exactly that of the referred columns and the metadata in the header and footer sections, as in Equation 16. The seek time depends on the number of retrieved columns (that might not be consecutively stored in disk), and their size. Equation 17 combines both components considering the weight of a read transfer as defined in Equation 13. 


$$
\begin{aligned}
& \operatorname{Used}_{\text {rows }}(\text { RowGroup })=\left\lfloor\frac{|M R|}{\operatorname{Used}_{R G}(\text { Hybrid })}\right\rfloor \\
& \operatorname{Size}(\operatorname{RefCols}) \quad=\operatorname{RefCols}(M R) *\left(\operatorname{Size}\left(\operatorname{Meta}_{Y C o l}\right)\right. \\
& \left.+\operatorname{Used}_{\text {rows }}(\text { RowGroup }) * \operatorname{Size}(\mathrm{Col})\right) \\
& \left.\operatorname{Size}\left(\text { Project }_{\text {hybrid }}\right) \quad=\operatorname{Size}\left(\text { Header }_{\text {hybrid }}\right)+\operatorname{Size}_{\left(\text {Footer }_{\text {hybrid }}\right)}\right) \\
& +\left(\operatorname{Size}(\operatorname{RefCols})+\operatorname{Size}\left(\operatorname{Meta}_{Y R G}\right)\right) \\
& \text { * } \operatorname{Used}_{R G}(\text { Hybrid }) \\
& +\left(\operatorname{Used}_{\text {chunks }}(\text { Hybrid }) * \operatorname{Size}\left(\text { Meta }_{\text {hybrid }}\right)\right) \\
& \text { Cost }_{\text {project }}(\text { Hybrid })=U \text { sed }_{\text {chunks }}\left(\text { Project }_{\text {hybrid }}\right) * W_{\text {ReadTransfer }} \\
& +\operatorname{Seek}(\text { Hybrid }) *\left(1-W_{\text {ReadTransfer }}\right)
\end{aligned}
$$

Hybrid layouts. They also natively support projection, and similarly to vertical layouts, we have to calculate its size to estimate the cost. However, hybrid layouts store data into multiple row groups. Therefore, we first need the row group size to estimate the projection size. As each row group contains a subset of rows, we estimate it as in Equation 18. Furthermore, Equation 19 gives the size of the columns used in the operation inside a group, which is then used in Equation 20 to estimate the overall projection size. Similar to the scan cost, hybrid layout also reads metadata separately for projection in each task, which we consider in the projection size. Hybrid layouts also have a seek cost to be considered, which depends on the number of row groups needed by the overall size of the file (not only of the result of the projection). Similar to previous cases, we can estimate the projection cost of hybrid layouts by appropriately weighting the transfer and seek times as in Equation 21.

\subsubsection{Selection}

It helps in fetching only some rows from disk (skipping others) to save some I/Os. As for projection, its cost depends on the support provided by each layout.

Horizontal and vertical layouts. They do not natively support this operation. They perform scan to bring all the data into memory and then filter them out based on the given predicate. Thus, their selection cost is the same as that of scan. 


$$
\begin{aligned}
& P(\text { RGSelected }) \quad=1-(1-S F)^{\text {Used }_{\text {rows }}(\text { RowGroup })} \\
& \text { Size }(\text { RowsSelected })=\left\lceil\frac{S F *|M R|}{U_{\text {sed }} \text { rows }(\text { RowGroup })}\right\rceil * \operatorname{Cols}(M R) \\
& *\left(\operatorname{Size}\left(\text { Meta }_{Y C o l}\right)+\operatorname{Used}_{\text {rows }}(\text { RowGroup }) * \operatorname{Size}(C o l)\right) \\
& \text { Used }_{R G}\left(\text { Select }_{\text {hybrid }}\right)= \begin{cases}U \operatorname{sed}_{R G}(\text { Hybrid }) & \\
* P(\text { RGSelected }) & \text { if Unsorted } \\
\left\lceil\frac{\text { Size }(\text { RowsSelected })}{\text { Size }(\text { RowGroup })}\right\rceil & \text { if Sorted }\end{cases} \\
& \operatorname{Size}\left(\text { Select }_{\text {hybrid }}\right) \quad=\operatorname{Size}\left(\text { Header }_{\text {hybrid }}\right)+\operatorname{Size}\left(\text { Footer }_{\text {hybrid }}\right) \\
& +\left(\operatorname{Used}_{R G}\left(\text { Select }_{\text {hybrid }}\right) * \operatorname{Size}(\text { RowGroup })\right) \\
& +\left(\operatorname{Used}_{\text {chunks }}(\text { Hybrid }) * \operatorname{Size}\left(\text { Meta }_{\text {hybrid }}\right)\right) \\
& \operatorname{Cost}_{\text {select }}(\text { Hybrid })=\operatorname{Used}_{\text {Chunks }}\left(\text { Select }_{\text {hybrid }}\right) * W_{\text {ReadTransfer }} \\
& +\operatorname{Seeks}\left(\text { Select }_{\text {hybrid }}\right) *\left(1-W_{\text {ReadTransfer }}\right)
\end{aligned}
$$

Hybrid layouts. They keep statistical information about data values in every column for every row group (typically, inside the header or footer sections). This helps in skipping some of the row groups that do not satisfy the predicate. Thus, the number of row groups to be read depends on the filtering condition and the sorting order of the column on which the selection is applied.

For unsorted columns, we can use the probability as in Equation 22 (borrowed from bitmap indexes [5]) to estimate the likelihood of any data in a row group satisfying the condition (i.e., a row group being fetched). In Equation 24, this probability is used to obtain the expected number of retrieved row groups. However, if a column is sorted, then we are using the Selectivity Factor $(S F)$ to estimate how much data is going to be read using Equation 23, which is later used in Equation 24 to calculate the fetched row groups for sorted columns (notice that all data fulfilling the condition is stored together if they are sorted on that column). Having the number of selected row groups, Equation 25 determines the size of a selection by adding up the total size of fetched row groups, metadata, header, and footer sections. As previously discussed about multiple reads of metadata in each task, we also consider this factor in the estimation of selection size.

Finally, this selection size can be used to estimate the total number of chunks and seeks as in Equations 2 and 3, which are then weighted as in Equation 26 to estimate the total selection cost. 


\section{Experiments}

In this section, we evaluate our approach and show the accuracy of our cost model for estimating the file sizes and the cost of scan, projection and selection for different storage formats. We choose representative storage formats from Apache Hadoop, the most popular distributed processing framework, because it is used in $59 \%$ of the enterprises to process big data, as shown in a survey from Cloudera [2]. In order to generate realistic data-intensive workflows, we rely on standard industry benchmarks. In our previous work [22], we used TPC-H for evaluating our rule-based approach given that TPC-H provides OLAP-like queries that are typically characterized by a low selectivity factor. To properly assess our cost-model, a broader range of analytical queries (i.e., typical reporting and data mining queries) are required. For this reason, we also leverage on TPC-DS for a more representative set of experiments.

Prior to conduct our experiments, we first instantiate our cost-model for Apache Hadoop. In HDFS, we can find several storage formats that follow the storage layouts discussed. Among them, we choose the most representative ones to show the effectiveness of our approach: SequenceFile (SeqFile) and Avro for horizontal layouts and Parquet for hybrid layouts. Appendix A contains all the details about the instantiation of these formats, including the file format size calculation and the required system variables. Note that, despite being included in Section 4 for the sake of completeness, we did not include any vertical layout, since those available for HDFS ended up being subsumed by hybrid ones and deprecated with time. Additionally, it is not possible to use hybrid layouts to mimic the behavior of vertical layouts, because the current implementations of hybrid layouts define a maximum limit on the size of row groups (i.e., 2GB), and do not allow creating different column groups (grouping different columns inside one vertical group and storing them rowwise). Finally, note also that for a fairer comparison, we are not considering encoding, which is available only in Parquet.

\subsection{Experimental setup}

Our experiments are performed on a 16-machines cluster ${ }^{15}$. Each machine has a Xeon E5-2630L v2 @2.40GHz CPU, 128GB of main memory and 1TB SATA3 of hard disk and runs Hadoop 2.6.2 and Pig 0.16.0 on Ubuntu 14.04 (64 bit). We have dedicated one machine for the HDFS name node and the remaining 15 machines for data nodes. We are using Apache Parquet 1.9.0, Avro 1.7.0 and elephant-bird $4.9^{16}$ for SeqFile.

\footnotetext{
${ }^{15} \mathrm{http}: / /$ www.ac.upc.edu/serveis-tic/altas-prestaciones

${ }^{16}$ https://github.com/twitter/elephant-bird
} 

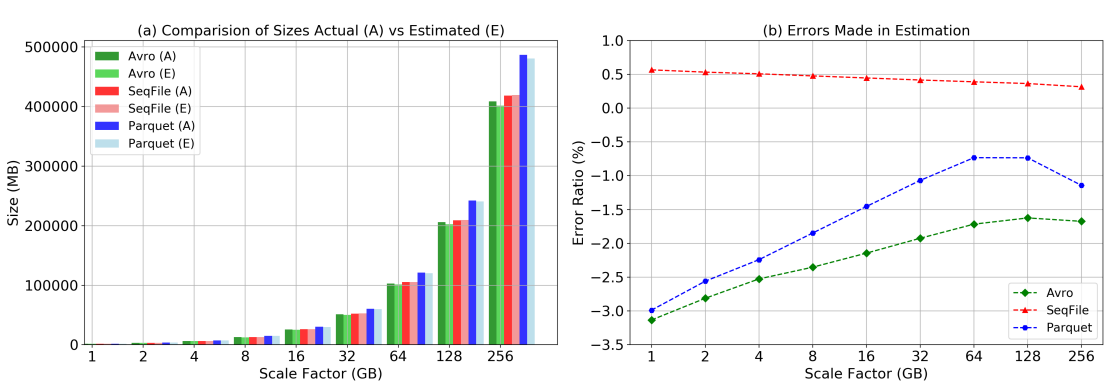

Fig. 9 Validating the size estimation

\subsection{Validation of file size estimations}

In this section, we are validating the accuracy of our size estimation by creating a synthetic MR (i.e., join of Lineitem and Part tables of TPC-H), and compare the actual size with the estimated one for each operation, namely scan, projection, and selection. We have chosen JOIN over other operations, because typically it is a computationally expensive operation and very common in modern DIFs. Figure 9 shows the results for scan operation on different scale factors. Figure 9a shows the results for the size, while Figure 9b shows the corresponding error rate for each studied format. We see that Avro and Parquet are slightly underestimated (up to $-3 \%$ error), while SeqFile is slightly overestimated (up to $0.5 \%$ ).
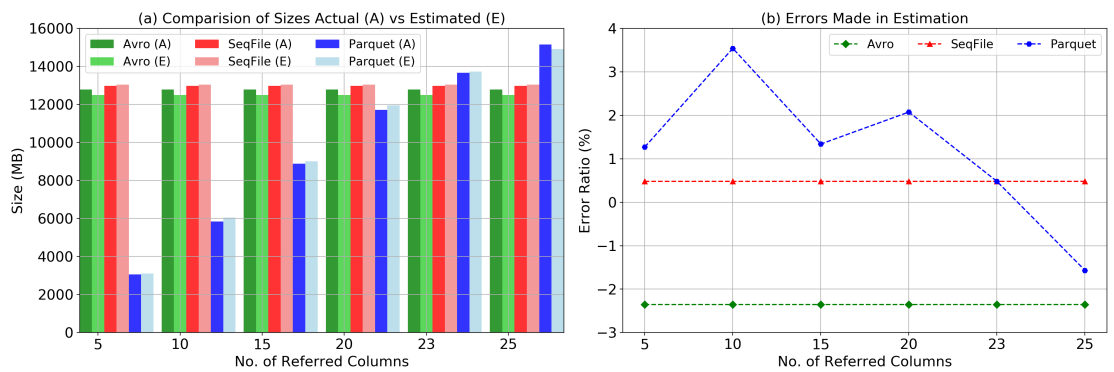

Fig. 10 Validating the projection cost model

Similarly, Figure 10 shows the results for validating our file size estimation after a projection. To do so, we read different number of columns, ranging from 5 to 25 , by executing 100 different runs, randomly selecting different columns on each run, over $8 \mathrm{~GB}$ and took the average of all runs. Figure 10a compares the actual and estimated size, and Figure 10b shows the percentage of error. SeqFile and Avro perform a scan for projection and their errors are the same as of the scan. However, Parquet has errors between $+4 \%$ to $-2 \%$, 
whose variance is due to variable column sizes (e.g., column with string data type), whereas we use average column size for all columns.
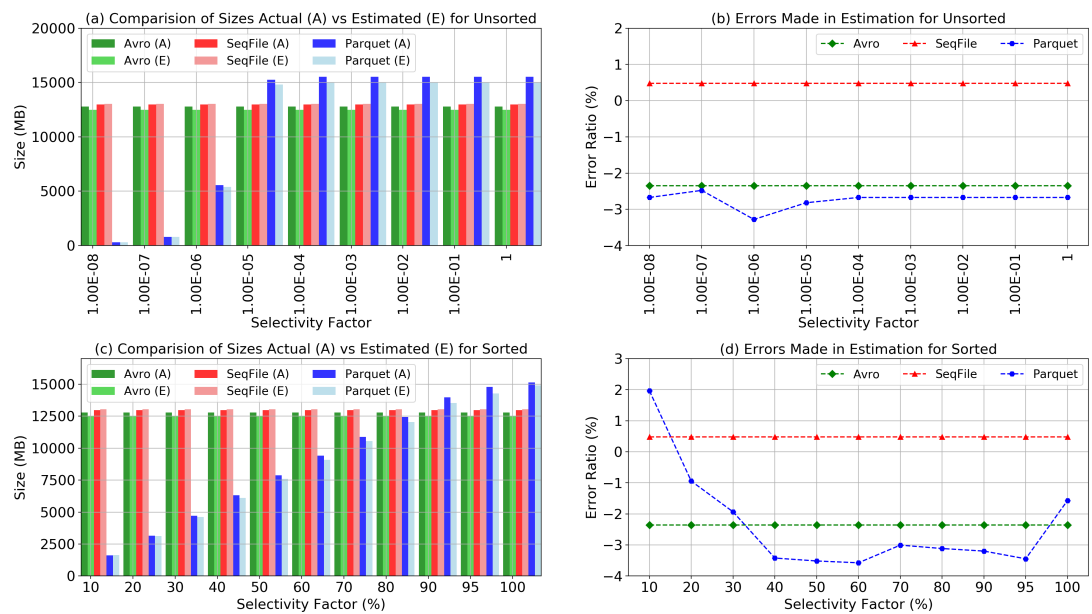

Fig. 11 Validating the selection cost model

Finally, Figure 11 validates the file size after a selection operation. For this experiment, we generate different selectivity factors. Also, since the sorting order of the filter column affects the reading, we are validating our results for both sorted and unsorted columns. Moreover, we repeated our experiments 100 times over $8 \mathrm{~GB}$ by randomly choosing different search values and took the average of all executions. Figures $11 \mathrm{a}$ and $11 \mathrm{~b}$ show the results of size estimations and errors in estimations, respectively, for unsorted columns. Observe that our cost model slightly underestimates the sizes (i.e., up to $-4 \%$ ). Moreover, the errors are more irregular when having small selectivity factors. This is due to the fact that when searching for few values, it is more difficult to find the exact row groups that contain those values. Figures $11 \mathrm{c}$ and $11 \mathrm{~d}$ show the results for sorted columns. Here our cost model for Parquet has errors in the range between $+2 \%$ to $-4 \%$ for the same reason discussed for unsorted columns.

All in all, the errors obtained in all our tests are rather small and consistent. Most importantly, we show next that these errors do not affect our prediction to choose the right storage format in all the experiments we conducted, since the estimated values still preserve the partial order among the actual values.

\subsection{Validation of file format choice}

In our previous work [22], we utilized TPC-H (i.e., OLAP-like workloads) for validating the accuracy of our heuristic rules and observed the importance of 
workload and data characteristics in selecting the most appropriate format. In this paper, we propose a cost model and validate it with both TPC-H and TPC-DS benchmarks. The goal is to cover a broader range of queries (i.e., broader workloads spanning reporting, OLAP and data mining). We have selected 6 out of 16 queries for TPC-H, and 16 out of 99 queries for TPCDS based on two main criteria: the selectivity factor (from $1 \%$ to $92 \%$ ), and the number of referred columns (from 3 to 66 ). All the selected queries were grouped based on these criteria. Then, representative queries are chosen with the goal of covering all the possible scenarios.

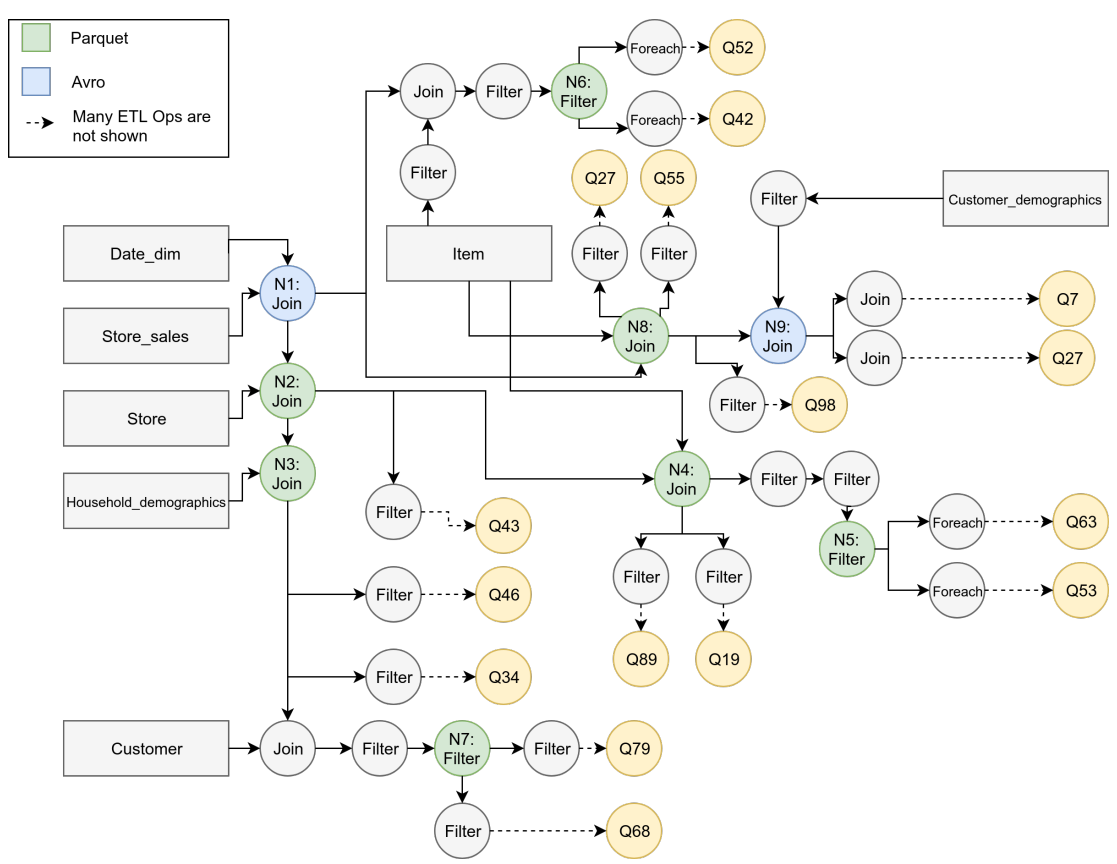

Fig. 12 DIW of 16 TPC-DS queries

In order to create a complex DIW, we used Quarry [18] to combine all TPC-DS queries into one integrated DIW as shown in Figure 12. To perform realistic experiments, we generate data with scale factors ranging from $1 \mathrm{~GB}$ to 256GB. In our experiments, ReStore (see Section 2.2) is used and nine nodes are selected to be materialized after applying both its aggressive and conservative heuristics. The aggressive heuristics decide to materialize the output of six joins and the conservative heuristics add three nodes resulting of filter operations. Additionally, we choose two metrics to analyze our approach, namely write cost (Section 4.1) and read cost (Section 4.2) for each materialized node. However, due to limitations in the native measurement of Hadoop performance, the charts corresponding to the read cost include also the exe- 
cution cost of the first operation right after reading the $M R$, since their costs cannot be decoupled.

Table 2 Materialized nodes with the statistics about their operations and chosen storage formats

\begin{tabular}{|c|c|c|c|c|c|}
\hline Node & Outgoing Operators & Rule-based & Trojan & Cost-based & Real Best Choice \\
\hline N1 & JOIN, JOIN & Avro & Avro & Avro & Avro \\
\hline $\mathrm{N} 2$ & $\begin{array}{l}\text { JOIN, JOIN, FILTER } \\
\text { (SF: 0.19) }\end{array}$ & Parquet & Avro & Avro & Avro \\
\hline N3 & $\begin{array}{l}\text { JOIN, FILTER (SF: } \\
0.59) \text {, FILTER (SF: } \\
0.01 \text { ) }\end{array}$ & Parquet & Avro & Avro & Avro \\
\hline $\mathrm{N} 4$ & $\begin{array}{l}\text { FILTER (SF: 0.03), } \\
\text { FILTER (SF: 0.2), } \\
\text { FILTER (SF: } 0.19)\end{array}$ & Parquet & Avro & Avro & Avro \\
\hline N5 & $\begin{array}{l}\text { FOREACH (Ref Cols: } \\
\text { 3), FOREACH (Ref } \\
\text { Cols: 3) }\end{array}$ & Parquet & Parquet & Parquet & Parquet \\
\hline N6 & $\begin{array}{l}\text { FOREACH (Ref Cols: } \\
\text { 15), FOREACH (Ref } \\
\text { Cols: 4) }\end{array}$ & Parquet & Avro & Parquet & Parquet \\
\hline N7 & $\begin{array}{l}\text { FILTER (SF: 0.13), } \\
\text { FILTER (SF: 0.92) }\end{array}$ & Parquet & Avro & Avro & Avro \\
\hline N8 & $\begin{array}{lll}\text { JOIN, } & \text { FILTER } & \text { (SF: } \\
0.19), & \text { FILTER } & \text { (SF: } \\
0.03), & \text { FILTER } & \text { (SF: } \\
0.01) & & \end{array}$ & Parquet & Avro & Avro & Avro \\
\hline N9 & JOIN, JOIN & Avro & Avro & Avro & Avro \\
\hline
\end{tabular}

*Projection is implemented as FOREACH in Apache PIG

\subsubsection{Rule-based approach}

Table 2 shows all nine nodes that have been materialized, together with their outgoing operators and storage formats decided by applying the heuristic rules from [22]. The rule-based column shows the choices made by our rule-based approach. The rationale behind these choices is as follows. Avro is chosen for N1 and N9, because the outgoing operators are joins, that use a scan access pattern, where Avro excels, as discussed in Section 2.1.1. For all other nodes, the rule-based approach is choosing Parquet. For Nodes N5 and N6, the outgoing edges contain FOREACH operations, where Parquet benefits from independent column storage. Nodes N4, N7 and N8 have FILTER operations in their outgoing edges, where Parquet can benefit from its native predicate push-down. Both FOREACH and FILTER operations only require a subset of data, and Parquet excels whenever a subset of data is read. Finally, nodes $\mathrm{N} 2$ and N3 have JOIN and FILTER as outgoing edges, and there would be different options to choose. However, for our rule-based approach Parquet is chosen, since, in case of several options available, it chooses the richest format providing more features. 


\subsubsection{Trojan cost-model}

The Trojan cost-model [16] provides cost formulas only for reading operations (i.e., scan). Since there is no estimation for writing operations and therefore, in our comparison we consider only the reading cost. For reading, Trojan considers the number of referred columns and always assumes a $100 \%$ selectivity factor. We executed the Trojan cost model on top of our scenario and the results obtained were as follows. Trojan selects Avro for all the nodes except N5. In N5, the number of referred columns are considered and therefore Parquet is predicted as the best choice. However, Trojan fails to predict N6 correctly. This is due to the fact that it assumes a high random reading cost (which in reality is not true, see Figure 14). For the other nodes, Trojan predicts correctly, however this is due to the fact that Parquet cannot filter when the selectivity factor is high. Thus, assumption of a high selectivity factor favors Avro. In a scenario with a low selectivity factor, Trojan would fail to correctly predict the storage layouts.

\subsubsection{Cost-based approach}

Note that Table 2 also shows some relevant collected statistics, such as the selectivity factor (SF) and the number of referred columns (Ref Cols), of the outgoing operators. It also shows the choices made by our cost-based approach. Here, we have not reported the estimated cost of our cost model, but we validated its choices with the actual executions, which confirms the accuracy of its predictions. Moreover, we have divided these nine nodes into three different color groups which are green, grey, and white. Green and grey groups contain nodes for which our rule-based approach works fine. Whereas, white group contains all the nodes for which our rule-based approach fails.
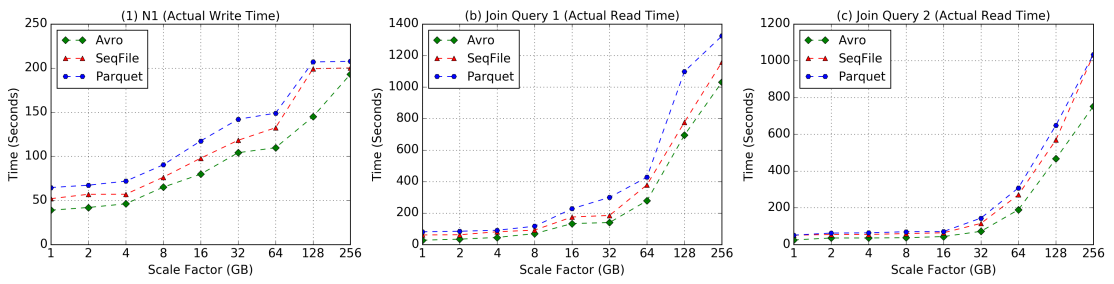

Fig. 13 Detailed experimentation conducted for node N1

Let us focus on N1 from the green group, for which the rule-based approach chooses the correct storage format (i.e., Avro). Figure 13 shows the actual write / read time of each storage format. It can be verified that the chosen layout (i.e., Avro) is always faster for both write and read operations.

Similarly, the rule-based approach also chooses the right storage format for grey group. Grey group contains nodes with projection operations. However, 

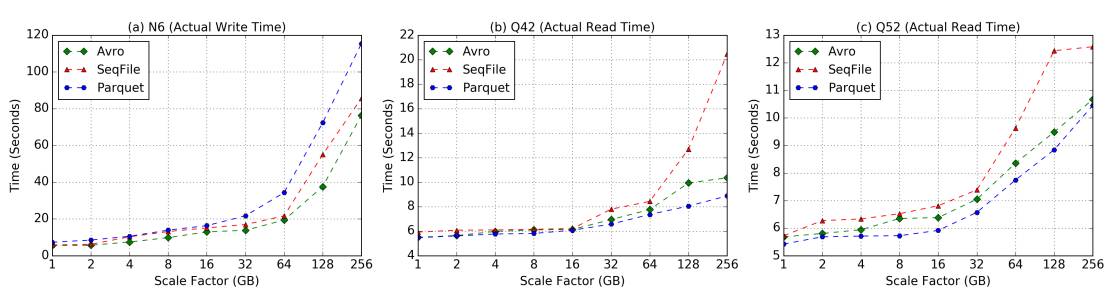

Fig. 14 Results for N6
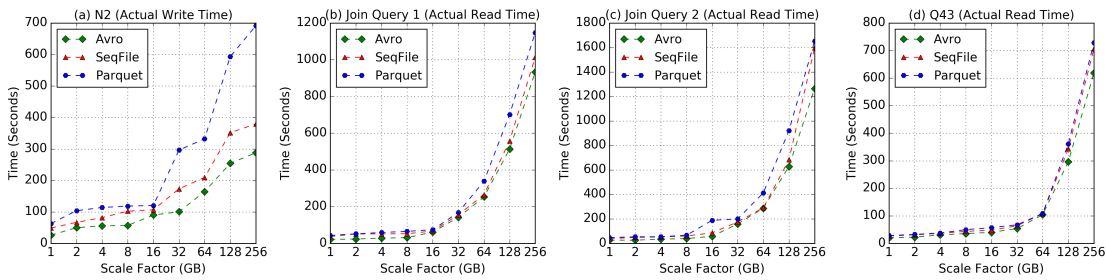

Fig. 15 Results for N2

the amount of data read is less than $70 \%$ and that's why it is better to use Parquet. Figure 14 shows the actual execution for N6. Figure 14a shows the actual execution time for both write and read operations. It can be seen that Parquet takes more time in writing (i.e., it writes more metadata), but its reading benefits compensate it as shown in Figures $14 \mathrm{~b}$ and $14 \mathrm{c}$ read time for Q42 and Q52, respectively.

On the other hand, the rule-based approach failed to choose the correct storage formats for the white group. All these nodes involve filter operations, where the amount of data to be read depends on the selectivity factor and all of them are greater than or equal to 0.1 (see Table 2). As already shown in Figure 11, different storage formats perform differently depending on the amount of data read. Therefore, since the rule-based approach does not leverage on statistics, the data volume to be read is not considered and it fails when choosing the right storage format. As it can be seen in Figure 11b, the predicate push-down mechanism implemented by Parquet is useless when the selectivity factor is greater than 1.0E-05 for unsorted columns. The rule-based approach always considers predicate push-down to be worth and thus still chooses Parquet. Oppositely, since our cost-based model considers the selectivity factor, it is able to select the right format for these nodes. For example, the results of N2 for the white group are shown in Figure 15, where the optimal choice is Avro, which takes less time than Parquet in both write and read operations. All the nodes of the white group follow the same trend and our cost-based approach successfully choses the right storage format.

In general, our cost model is able to decide the right format in all cases shown in Table 2, because it considers the amount of data read (which in this case is determined by the format file size and the selectivity factor of the operation), which actually depends on two operations, namely projection and 


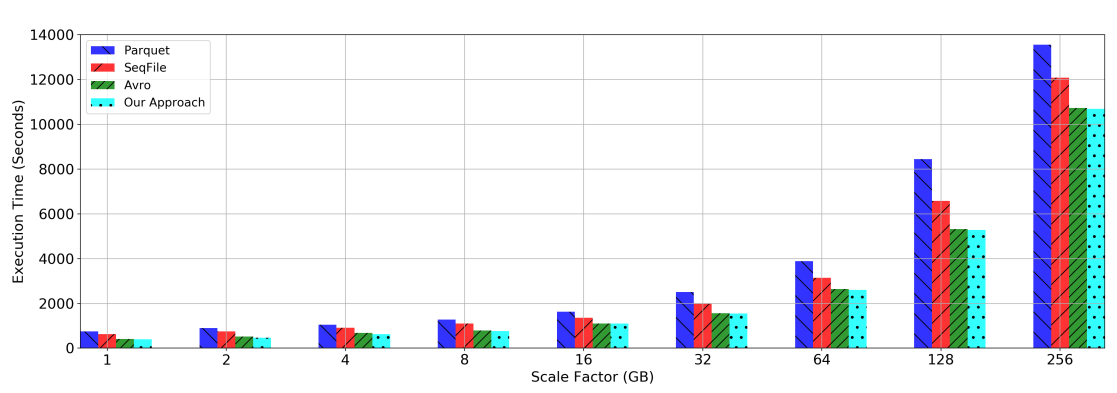

Fig. 16 Single Fixed Format vs Our Approach for TPC-DS

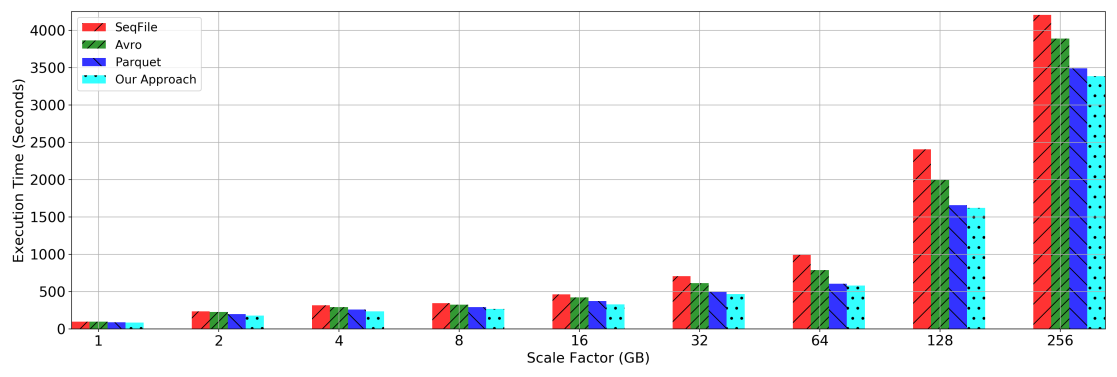

Fig. 17 Single Fixed Format vs Our Approach for TPC-H

selection. Figure 16 compares our approach with a typical approach materializing all chosen $M R$ with a fixed format (i.e., always SequenceFile, Parquet or Avro). It shows the overall execution time of the DIW when using a single fixed format for $M R$ with regard to a dynamic choice of the format based on our cost model. Our approach on average provides 1.6x speed up over fixed Parquet, 1.34x speedup over fixed SequenceFile, 1.03x speedup over fixed Avro and, in the average, it provides 1.33x speedup for TPC-DS.

Note that in TPC-DS, our cost model favors Avro, and this is due to the fact that the chosen $M R$ have subsequent operations with high selectivity factors. In contrast, when we changed the workload to TPC-H, the cost model recommends Parquet in the majority of materialized nodes, due to the low selectivity queries. The overall results of TPC-H are shown in Figure 17. Observe that, for TPC-H, our approach on average provides $1.32 \mathrm{x}$ speedup over fixed SequenceFile, 1.19x speedup over fixed Avro, 1.04x speedup over fixed Parquet and overall, it provides 1.18x speedup.

Note that, a given kind of workload may favor a certain format. However, a system should be able to adapt to different workloads as we saw when comparing TPC-DS (scan-based favors Avro) and TPC-H (selection-based and projection-based favor Parquet). In conclusion, our cost model is capable of choosing the appropriate storage format for different workloads, which always leads to improvements in query execution time. 


\section{Related Work}

In this section, we first discuss the related work on choosing different storage layouts. Then, we discuss in detail the existing cost models for distributed processing frameworks.

\subsection{Use of different storage layouts}

The fixed layout problem is already identified by the research community and many solutions have been proposed. The existing solutions allow using multiple layouts together. For instance, the in-memory DBMS SAP HANA [10] uses horizontal and vertical layouts for On-line Transaction Processing (OLTP) and On-line Analytical Processing (OLAP) workloads, respectively. In a similar way, in DB2 [24] horizontal and vertical layouts can be used for the same table-space. However, these layouts are fixed and non-modifiable at runtime.

On the other hand, there are also solutions that consider workloads in order to decide for the most suitable layout. These systems work in multi-database environments. Polybase [7] for instance, is a system that uses both Hadoop cluster and DBMS for data storage. Based on the workloads, it dynamically decides which is the best solution. According to this decision, it also moves the data from one system to another for the execution of the queries. This solution focuses on utilizing the processing power of the Hadoop cluster and it always uses a horizontal layout to store data on Hadoop.

Similar to Polybase, there is a hybrid system [13], which can read raw files directly and it can choose the physical layout of the data in the DBMS based on the input queries. However, they propose to keep multiple copies of the same data in different formats, which is not always feasible, especially when the size of the data is huge.

In addition, there are two systems $[9,25]$ that store the data inside different storage engines by taking into account the data access patterns. These systems work like mediators and analyze the characteristics of the data to then route them to the most suitable storage engine. In [9], the system requires training in order to take the right decision in choosing the best storage engine for queries. Furthermore, this training runs every query in all available systems to see which system is good for most of the queries. Hence, this requires extra processing and adds extra cost. In [25], the solution relies on annotations which are defined by the user during the requirements definition process of an application. These annotations help the mediator to decide where to store the application data. The annotations however cannot be defined at run-time. Moreover, this solution mainly focuses on choosing a storage engine according to the application requirements without considering the physical storage layout.

There is a solution, $\mathrm{H} 2 \mathrm{O}$ [1], that can dynamically decide the physical layout of the data based on the current workload. However, it only supports 
vertical layouts by creating different column groups. Moreover, as described there, creating column groups is a NP-hard problem and it is not feasible for a table which has many columns. Additionally, WWHow [17] proposes a data layer which is independent of the physical storage. This layer enables an adaptable physical storage engine by analyzing the application needs. However, they are considering general storage systems such as file-systems, databases and cloud storages without considering the physical layouts of the data in them. Moreover, once decided, the storage system remains fixed. [3] proposes a caching approach for nested data (i.e., JSON). It helps to keep more frequent used data in cache by storing in appropriate layout, according to the running workload. This work also supports our hypothesis to use different layouts for different type of workloads. However, it is limited to only nested data and not applicable to other scenarios.

\subsection{Existing cost models}

Trojan [16] is an adaptable column storage for Hadoop that handles different types of workloads. It takes advantage of the data replication feature of HDFS, and analyzes the workload access patterns to store different column groups on each replica. Then, it routes every query to the node where the replica of the data has the most suitable layout for that particular query. However, it considers only the vertical storage layouts and ignores scan based workloads. They also proposed a cost model for different storage layouts, but their cost model considers only the scan operation.

Furthermore, there is also a cost model for Hadoop jobs, Starfish [12], whose cost model helps to measure execution time. It considers different parameters to calculate the execution time, and it can help to design a cost-based optimizer. However, it does not consider different storage layouts.

Finally, [4] helps in reducing the seek cost in a wide table by storing the columns in an appropriate order based on the access patterns. This approach helps to reduce the disk cost and, overall, it reduces the execution cost of different queries. However, it considers only hybrid layouts in their study and it provides a cost model only for estimating the seek cost.

\section{Conclusion}

Modern analytical workloads involve different types of queries in which a fixed storage format for MRs does not guarantee the best performance. Additionally, the currently available solutions have not considered at the same time choosing both MRs and the types of the storage layouts to be used for their storage. They consider these problems separately and therefore fail to provide an optimal solution. We explicitly focus on choosing the storage layouts for MRs and propose a whole process-cycle. Our proposed approach uses any existing solution to choose MRs and after deciding which MRs in a data intensive workflow to store, it chooses the best storage format, which improves 
performance, by analyzing their access patterns. Overall, this reduces the load time and, in general, the total workflow execution time. We have implemented our generic cost-based model for Hadoop and instantiated on different storage formats to show its effectiveness. Our evaluation results show the benefits of our approach and support our hypothesis that $M R$ should be persistent by considering the best storage format.

Acknowledgements This research has been funded by the European Commission through the Erasmus Mundus Joint Doctorate "Information Technologies for Business Intelligence Doctoral College" (IT4BI-DC)

\section{References}

1. Alagiannis, I., Idreos, S., Ailamaki, A.: H2O: a hands-free adaptive store. In: International Conference on Management of Data, SIGMOD 2014, Snowbird, UT, USA, June 22-27, 2014, pp. 1103-1114 (2014)

2. Atscale: Big data maturity survey. Cloudera (2016)

3. Azim, T., Karpathiotakis, M., Ailamaki, A.: Recache: Reactive caching for fast analytics over heterogeneous data. PVLDB 11(3), 324-337 (2017)

4. Bian, H., Yan, Y., Tao, W., Chen, L.J., Chen, Y., Du, X., Moscibroda, T.: Wide table layout optimization based on column ordering and duplication. In: Proceedings of the 2017 ACM International Conference on Management of Data, SIGMOD Conference 2017, Chicago, IL, USA, May 14-19, 2017, pp. 299-314 (2017)

5. Cardenas, A.F.: Analysis and performance of inverted data base structures. Commun. ACM 18(5), 253-263 (1975)

6. Chen, Y., Alspaugh, S., Katz, R.H.: Interactive analytical processing in big data systems: A cross-industry study of mapreduce workloads. PVLDB 5(12), 1802-1813 (2012)

7. DeWitt, D.J., Halverson, A., Nehme, R.V., Shankar, S., Aguilar-Saborit, J., Avanes, A., Flasza, M., Gramling, J.: Split query processing in polybase. In: Proceedings of the ACM SIGMOD International Conference on Management of Data, SIGMOD 2013, New York, NY, USA, June 22-27, 2013, pp. 1255-1266 (2013)

8. Elghandour, I., Aboulnaga, A.: Restore: Reusing results of mapreduce jobs. PVLDB $\mathbf{5}(6), 586-597(2012)$

9. Elmore, A.J., Duggan, J., Stonebraker, M., Balazinska, M., Çetintemel, U., Gadepally, V., Heer, J., Howe, B., Kepner, J., Kraska, T., Madden, S., Maier, D., Mattson, T.G., Papadopoulos, S., Parkhurst, J., Tatbul, N., Vartak, M., Zdonik, S.: A demonstration of the bigdawg polystore system. PVLDB 8(12), 1908-1911 (2015)

10. Färber, F., Cha, S.K., Primsch, J., Bornhövd, C., Sigg, S., Lehner, W.: SAP HANA database: data management for modern business applications. SIGMOD Record 40(4), 45-51 (2011)

11. Floratou, A., Patel, J.M., Shekita, E.J., Tata, S.: Column-oriented storage techniques for mapreduce. PVLDB 4(7), 419-429 (2011)

12. Herodotou, H., Babu, S.: Profiling, what-if analysis, and cost-based optimization of mapreduce programs. PVLDB 4(11), 1111-1122 (2011)

13. Idreos, S., Alagiannis, I., Johnson, R., Ailamaki, A.: Here are my data files. here are my queries. where are my results? In: CIDR 2011, Fifth Biennial Conference on Innovative Data Systems Research, Asilomar, CA, USA, January 9-12, 2011, Online Proceedings, pp. 57-68 (2011)

14. Jindal, A., Karanasos, K., Rao, S., Patel, H.: Selecting subexpressions to materialize at datacenter scale. PVLDB 11(7), 800-812 (2018)

15. Jindal, A., Qiao, S., Patel, H., Yin, Z., Di, J., Bag, M., Friedman, M., Lin, Y., Karanasos, K., Rao, S.: Computation reuse in analytics job service at microsoft. In: SIGMOD Conference, pp. 191-203 (2018) 
16. Jindal, A., Quiané-Ruiz, J., Dittrich, J.: Trojan data layouts: right shoes for a running elephant. In: ACM Symposium on Cloud Computing in conjunction with SOSP 2011, SOCC '11, Cascais, Portugal, October 26-28, 2011, p. 21 (2011)

17. Jindal, A., Quiané-Ruiz, J., Dittrich, J.: Wwhow! freeing data storage from cages. In: CIDR 2013, Sixth Biennial Conference on Innovative Data Systems Research, Asilomar, CA, USA, January 6-9, 2013, Online Proceedings (2013)

18. Jovanovic, P., Romero, O., Simitsis, A., Abelló, A.: Incremental consolidation of dataintensive multi-flows. IEEE Trans. Knowl. Data Eng. 28(5), 1203-1216 (2016)

19. Kalavri, V., Shang, H., Vlassov, V.: m2r2: A framework for results materialization and reuse in high-level dataflow systems for big data. In: 16th IEEE International Conference on Computational Science and Engineering, CSE 2013, December 3-5, 2013, Sydney, Australia, pp. 894-901 (2013)

20. Laga, A., Boukhobza, J., Koskas, M., Singhoff, F.: Lynx: a learning linux prefetching mechanism for SSD performance model. In: NVMSA, pp. 1-6 (2016)

21. Munir, R.F., Nadal, S., Romero, O., Abelló, A., Jovanovic, P., Thiele, M., Lehner, W.: Intermediate results materialization selection and format for data-intensive flows (accepted). Fundamenta Informaticae (2018)

22. Munir, R.F., Romero, O., Abelló, A., Bilalli, B., Thiele, M., Lehner, W.: Resilientstore: A heuristic-based data format selector for intermediate results. In: Model and Data Engineering - 6th International Conference, MEDI 2016, Almería, Spain, September 21-23, 2016, Proceedings, pp. 42-56 (2016)

23. Nykiel, T., Potamias, M., Mishra, C., Kollios, G., Koudas, N.: Mrshare: Sharing across multiple queries in mapreduce. Proc. VLDB Endow. 3(1-2), 494-505 (2010)

24. Raman, V., Attaluri, G.K., Barber, R., Chainani, N., Kalmuk, D., KulandaiSamy, V., Leenstra, J., Lightstone, S., Liu, S., Lohman, G.M., Malkemus, T., Müller, R., Pandis, I., Schiefer, B., Sharpe, D., Sidle, R., Storm, A.J., Zhang, L.: DB2 with BLU acceleration: So much more than just a column store. PVLDB 6(11), 1080-1091 (2013)

25. Schaarschmidt, M., Gessert, F., Ritter, N.: Towards automated polyglot persistence. In: Datenbanksysteme für Business, Technologie und Web (BTW), 16. Fachtagung des GIFachbereichs "Datenbanken und Informationssysteme" (DBIS), 4.-6.3.2015 in Hamburg, Germany. Proceedings, pp. 73-82 (2015)

26. Shvachko, K.V.: Hdfs scalability: the limits to growth. Login 35(2), 6-16 (2010)

27. Silva, Y.N., Larson, P.A., Zhou, J.: Exploiting common subexpressions for cloud query processing. In: Proceedings of the 2012 IEEE 28th International Conference on Data Engineering, pp. 1337-1348 (2012)

\section{A}

This appendix shows the file sizes for the three considered HDFS file formats, together with the system variables with their values according to our testbed. Table 3 lists all the system variables. They are divided in three categories. First category has the variables related to disk which are important to calculate the reading and writing cost. Additionally, second category has variables for network to calculate the transfer cost, since Hadoop writes multiple copy of data for fault tolerance purpose and this involves writing to other nodes. For this writing, it needs to transfer data, and it is important in calculating the overall write cost. Final category lists the variables related to the configuration of our Hadoop cluster.

\begin{tabular}{|c|c|c|c|c|c|c|c|c|c|c|}
\hline $\begin{array}{c}\text { Version } \\
\text { (4 Bytes) }\end{array}$ & $\begin{array}{c}\text { Key Class } \\
\text { (4 Bytes) }\end{array}$ & $\begin{array}{c}\text { Value Class } \\
\text { (4 Bytes) }\end{array}$ & $\begin{array}{c}\text { Record } \\
\text { Compression } \\
\text { (1 Byte) }\end{array}$ & $\begin{array}{c}\text { Block } \\
\text { Compression } \\
\text { (1 Byte) }\end{array}$ & $\begin{array}{c}\text { Sync Marker } \\
\text { (16 Bytes) }\end{array}$ & $\begin{array}{c}\text { Record } \\
\text { Lenth } \\
\text { (4 Bytes) }\end{array}$ & $\begin{array}{c}\text { Key Length } \\
\text { (4 Bytes) }\end{array}$ & $\begin{array}{c}\text { Key } \\
\text { (Long) }\end{array}$ & $\begin{array}{c}\text { Values } \\
\text { (Dynamic) }\end{array}$ & $\begin{array}{c}\text { Sync Marker } \\
\text { (16 Bytes) }\end{array}$ \\
\hline
\end{tabular}

Fig. 18 Physical file format of SequenceFile 
Table 3 System variables with their values according to our testbed

\begin{tabular}{|c|c|c|}
\hline \multicolumn{3}{|c|}{ Variables for Disk } \\
\hline$B W_{d i s k}$ & Disk bandwidth & $\begin{array}{l}1.3 \times 10^{8} \\
\text { bytes } / \text { second }\end{array}$ \\
\hline Size $($ Block $)$ & Disk block size & $8.0 \times 10^{3}$ bytes \\
\hline Time $_{\text {seek }}$ & Disk random seek time & $\begin{array}{l}5.0 \times 10^{-3} \text { sec- } \\
\text { onds }\end{array}$ \\
\hline Time $_{\text {rotation }}$ & Disk rotation time & $\begin{array}{l}4.17 \times 10^{-6} \\
\text { seconds }\end{array}$ \\
\hline \multicolumn{3}{|c|}{ Variables for Network } \\
\hline$B W_{n e t}$ & Network bandwidth & $\begin{array}{l}1.25 \times 10^{8} \\
\text { bytes/second }\end{array}$ \\
\hline \multicolumn{3}{|c|}{ Variables for Hadoop } \\
\hline Size $($ Chunk $)$ & HDFS block size & $\begin{array}{l}1.28 \times 10^{8} \\
\text { bytes }\end{array}$ \\
\hline Size(Buffer) & Buffer size & $6.4 \times 10^{4}$ bytes \\
\hline$R$ & Replication factor & 3 \\
\hline$p$ & $\begin{array}{l}\text { Probability of accessed } \\
\text { replica being local [16] }\end{array}$ & 0.97 \\
\hline
\end{tabular}

A.1 SequenceFile (SeqFile) format

Table 4 Sizes of SeqFile according to our testbed

\begin{tabular}{|c|c|c|}
\hline \multicolumn{3}{|c|}{ Variables for SeqFile } \\
\hline Size $\left(\right.$ Header $\left._{\text {SeqFile }}\right)$ & Header size of SeqFile & 30 \\
\hline Size(RecordLength) & Fixed field & 4 \\
\hline Size (KeyLength $)$ & Number of bytes for key & 4 \\
\hline $\operatorname{Size}\left(\right.$ Meta $\left._{S C o l}\right)$ & $\begin{array}{l}\text { Number of bytes for user- } \\
\text { defined separator per column }\end{array}$ & 1 \\
\hline Size(SyncMarker $)$ & Size of sync marker & 16 \\
\hline Size $($ SyncBlock $)$ & $\begin{array}{l}\text { Number of bytes between sync } \\
\text { markers }\end{array}$ & 2,000 \\
\hline Size $\left(\right.$ Footer $\left._{\text {SeqFile }}\right)$ & Footer size & 0 \\
\hline
\end{tabular}

SeqFile ${ }^{17}$ is introduced in 2009 to improve the performance of MapReduce framework. It is used to store the temporary output of map phases as compressed to reduce I/Os. Moreover, it is also splittable which is ideal for processing in parallel. It considers a special type of horizontal layout, which stores data in the form of key-value pairs. Figure 18 shows its structure and Table 4 shows the specific variables of SeqFile with their values.

\footnotetext{
${ }^{17}$ https://wiki.apache.org/hadoop/SequenceFile
} 


$$
\begin{aligned}
& \operatorname{Size}\left(\text { Row }_{\text {SeqFile }}\right) \quad=\operatorname{Size}(\text { RecordLength }) \\
& + \text { Size (KeyLength) } \\
& +\operatorname{Size}(\mathrm{Col}) * \operatorname{Cols}(\mathrm{MR}) \\
& +\operatorname{Size}\left(M_{\text {eta }} a_{S o l}\right) *(\operatorname{Cols}(M R)-2) \\
& \operatorname{Size}\left(\text { TotalRows }_{\text {SeqFile }}\right)=\operatorname{Size}\left(\text { Row }_{\text {SeqFile }}\right) *|M R| \\
& \operatorname{Size}\left(\text { Meta }_{\text {SBody }}\right) \quad=\left\lceil\frac{\text { Size }\left(\text { TotalRows }_{\text {SeqFile }}\right)}{\text { Size }(\text { SyncBlock })}\right\rceil \\
& \text { * Size(SyncMarker) } \\
& \text { Size }\left(\text { Body }_{\text {SeqFile }}\right) \quad=\text { Size }\left(\text { TotalRows }_{\text {SeqFile }}\right) \\
& +\operatorname{Size}\left(\operatorname{Meta}_{S B o d y}\right)
\end{aligned}
$$

To instantiate from our generic cost model, we need to estimate the sizes of header, body and footer sections. The header section of SeqFile has a fixed size, so we define it as a constant. To estimate body size, we need to calculate row and metadata sizes. SeqFile divides each row into a key-value pair and stores one column into the key, and the remaining columns into the value by using a user-defined separator. Thus, it has two types of metadata: one is used to separate values and another to make blocks for parallel processing. Then, the size of a row is compound of some fields of fixed size (i.e., record and key lengths) together with the corresponding key-value pair as shown in Figure 18, containing all user columns (notice that we need two less user-defined separators than columns, because the key is managed by the file format itself). Equation 27 is estimating this size (i.e., a row for SeqFile), which is later used in Equation 28 to estimate the size of all key-value pairs. Equation 29 calculates the overhead of block-related metadata (i.e., sync markers), which SeqFile introduces at fixed intervals. Finally, Equation 30 simply adds the size of key-value pairs and metadata, which allows in turn to obtain the total size of SeqFile using Equation 1 with an empty footer section.

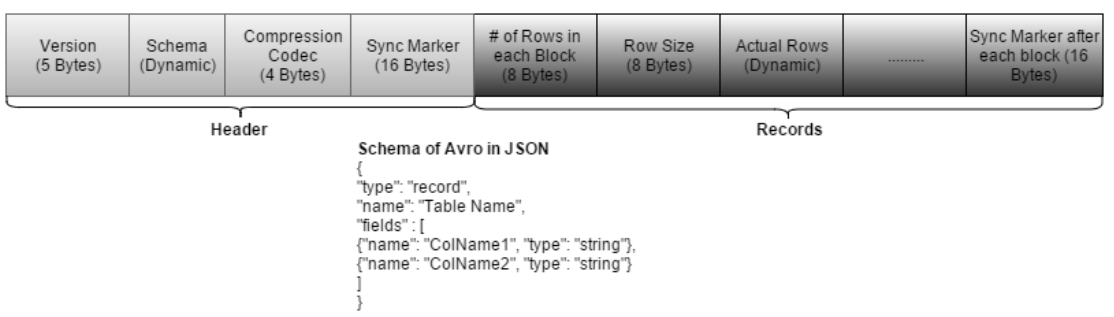

Fig. 19 Physical file format of Avro

\section{A.2 Avro format}

Apache Avro ${ }^{18}$ is a language-neutral data serialization system. It means Avro can be written in one language and can be read in another language without changing the code. This support

${ }^{18}$ https://www.tutorialspoint.com/avro/avro_tutorial.pdf 
Table 5 Sizes of Avro according to our testbed

\begin{tabular}{|c|c|c|}
\hline \multicolumn{3}{|c|}{ Variables for Avro } \\
\hline Size $($ Version $)$ & Version of Avro & 5 \\
\hline Size (Codec) & Compression codec & 4 \\
\hline Size(SyncMarker) & Size of sync marker & 16 \\
\hline Size (ColSchema) & $\begin{array}{l}\text { Size of schema information per } \\
\text { column }\end{array}$ & $\sim 30$ bytes \\
\hline $\operatorname{Size}\left(B_{\text {avro }}\right)$ & Block size of Avro & 4,000 \\
\hline Size $\left(\right.$ Meta $\left._{A R o w}\right)$ & Meta information for each row & 8 \\
\hline $\operatorname{Size}\left(\right.$ Meta $\left._{A B l o c k}\right)$ & $\begin{array}{l}\text { Meta information for each } \\
\text { block }\end{array}$ & 8 \\
\hline Size $\left(\right.$ Footer $\left._{\text {Avro }}\right)$ & Footer size & 0 \\
\hline
\end{tabular}

is provided by the schema information which Avro stores as a meta information. Moreover, it is also compressible and splitable. It is a horizontal layout and Figure 19 sketches its physical structure. Moreover, there are specific variables for Avro which are given in Table 5. The data schema is stored in a header section of variable length. Similarly, the size of body is also variable and it depends on the number of rows in an IR.

$$
\begin{aligned}
& \operatorname{Size}\left(\text { Header }_{\text {avro }}\right) \quad=\operatorname{Size}(\text { Version }) \\
& +\operatorname{Cols}(M R) * \operatorname{Size}(\text { ColSchema }) \\
& + \text { Size }(\text { Codec })+\text { Size }(\text { SyncMarker }) \\
& \operatorname{Size}\left(\text { TotalRows }_{\text {Avro }}\right)=\left(\operatorname{Size}(\operatorname{Row})+\operatorname{Size}\left(\operatorname{Meta}_{A R o w}\right)\right) *|M R| \\
& \operatorname{Size}\left(\text { Meta }_{A B o d y}\right)=\left(\text { Meta }_{A B l o c k}+\operatorname{Size}(\text { SyncMarker })\right) \\
& \text { * }\left\lceil\frac{\text { Size }\left(\text { TotalRows }_{\text {Avro }}\right)}{\operatorname{Size}\left(B_{\text {Avro }}\right)}\right\rceil \\
& \operatorname{Size}\left(\text { Body }_{\text {avro }}\right) \quad=\operatorname{Size}(\text { TotalRows })+\operatorname{Size}\left(\text { Meta }_{A B o d y}\right)
\end{aligned}
$$

Header section of Avro contains meta information corresponding to the schema of the data in the form a JSON. Given that the size of the schema is orders of magnitude smaller that data, we estimate it as a constant per column. Considering also the version and codec information, the overall header size is calculated by Equation 31. Following the horizontal layout, Avro adds metadata to each row, which is considered in Equation 32 to estimate the size of a row. Moreover, it also adds extra metadata in the body for every block. Thus, Equation 33 is calculating the total size of metadata by multiplying the number of blocks by the size of sync marker and that of counter for the number of rows in the block. Finally, Equation 34 is used to calculate the body size, which allows in turn to obtain the total size of Avro using Equation 1 with an empty footer section.

\section{A.3 Parquet format}

Apache Parquet ${ }^{19}$ is introduced in 2013 to provide hybrid layout support for Hadoop echosystem. It divides data horizontally into row groups, whereas each row group is further divided vertically to store columns separately, as sketched in Figure 20. Additionally, it also divides each vertical partition into multiple pages. Moreover, it also stores the schema and statistical information about the data as meta information in the footer section. All variables specific to Parquet are listed in Table 6.

\footnotetext{
${ }^{19} \mathrm{http}: / /$ parquet.apache.org
} 


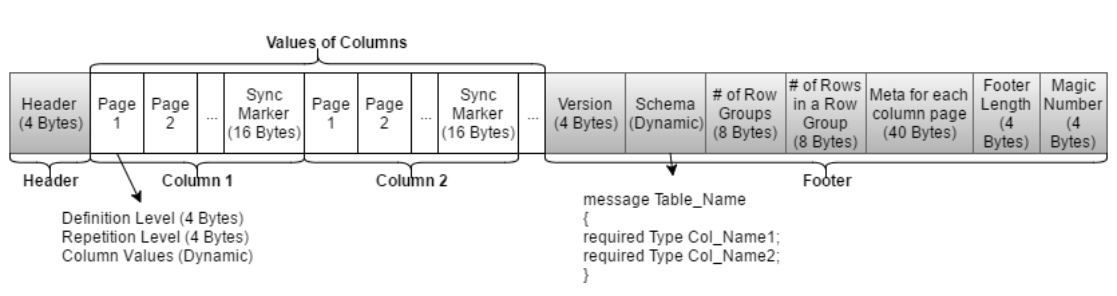

Fig. 20 Physical file format of Parquet

Table 6 Sizes of Parquet according to our testbed

\begin{tabular}{|c|c|c|}
\hline \multicolumn{3}{|c|}{ Variables for Parquet } \\
\hline Size $\left(\right.$ Header $\left._{\text {parquet }}\right)$ & Size of header & 4 \\
\hline Size (DefinitionLevel) & Size of definition level & 4 \\
\hline Size(RepetitionLevel) & Size of repetition level & 4 \\
\hline Size(RowCounter) & Size of number of rows & 8 \\
\hline Size(SyncMarker) & Size of sync marker & 16 \\
\hline Size(Version) & Version in footer & 4 \\
\hline Size (ColSchema) & $\begin{array}{l}\text { Size of schema informa- } \\
\text { tion per column }\end{array}$ & 30 bytes \\
\hline $\operatorname{Size}\left(\right.$ Meta $\left._{P C o l}\right)$ & $\begin{array}{l}\text { Size of columns meta } \\
\text { data for storing statis- } \\
\text { tical information }\end{array}$ & 40 \\
\hline Size(MagicNumber $)$ & Magic number in footer & 4 \\
\hline Size (Footer Length) & Footer length in footer & 4 \\
\hline Size(RowGroup) & Layout row group size & $\begin{array}{l}1.28 \times 10^{8} \\
\text { bytes }\end{array}$ \\
\hline Size(Page) & Layout page size & $\begin{array}{l}1.05 \times 10^{6} \\
\text { bytes }\end{array}$ \\
\hline
\end{tabular}

$$
\begin{aligned}
& \operatorname{Used}_{\text {pages }}\left(\text { RowGroup }_{\text {parquet }}\right)=(\operatorname{Size}(\mathrm{Col}) \\
& \text { * Used } \operatorname{siws}_{\text {row }}(\text { Row Group parquet }) \\
& + \text { Size }(\text { SyncMarker })) \\
& * \frac{\operatorname{Cols}(M R)}{\text { Size }(\text { Page })} \\
& \text { Size }\left(\text { Body } y_{\text {parquet }}\right) \quad=(((\text { Size }(\text { DefinitionLevel }) \\
& + \text { Size(RepetitionLevel) } \\
& + \text { Size(Page)) } \\
& \text { * } \left.\operatorname{Used}_{\text {pages }}(\text { RowGroup parquet })\right) \\
& + \text { Size(RowCounter) } \\
& + \text { Size (SyncMarker })) \\
& \text { * } U \operatorname{sed}_{R G} \text { (Parquet) } \\
& \operatorname{Size}\left(\text { Footer }_{\text {parquet }}\right) \quad=\operatorname{Size}(\text { Version }) \\
& +\operatorname{Size}(\text { ColSchema }) * \operatorname{Cols}(M R) \\
& + \text { Size(MagicNumber) } \\
& + \text { Size(FooterLength) } \\
& +\operatorname{Used}_{R G}(\text { Parquet }) * \operatorname{Size}\left(\text { Meta }_{P C o l}\right) \\
& *\left(1+\operatorname{Used}_{\text {pages }}\left(\text { RowGroup }_{\text {parquet }}\right)\right)
\end{aligned}
$$


The header section of Parquet has a fixed size, as stated in Table 6. To estimate the body size, we first need to estimate the total number of row groups (i.e., Equation 9) and the total rows per row group (i.e., Equation 18). Moreover, we need to be aware that Parquet stores every individual column divided it into multiple pages, whose number which is estimated by Equation 35 per row group. Next, we are calculating the body size of Parquet using Equation 36, by considering metadata for each page (namely definition level and repetition level), and for every row group (namely counter of rows per row group and sync marker).

Finally, we calculate the footer size by approximating the size the of the schema, sketched in Figure 20, by a constant amount of bytes per column. Moreover, Parquet also stores statistical information about columns in the Footer section for both row groups and data pages. Equation 37 uses all these values together to calculate overall size of footer. Then, total size of Parquet is obtained by adding the header, body and footer sections, as defined in Equation 1. 